\title{
La intervención en competencia social en estudiantes con TDAH: Un estudio de revisión
}

\author{
Gisela Rojo-Amato. Universitat Oberta de Catalunya \\ Raúl Tárraga-Mínguez. Universidad de Valencia \\ Pilar Sanz-Cervera. Universidad de Valencia
}

Recepción: 6 de septiembre de 2016 | Revisión: 15 de septiembre de 2016 | Aceptado: 7 diciembre de 2016
Correspondencia: Pilar Sanz-Cervera | Email: Pilar.Sanz-Cervera@uv.es | ORCID: 0000-0001-6919-6150
Citar: Rojo-Amato, G., Tarraga-Minguez, R. y Sanz-Cervera, P. (2016). La intervención en competencia social en estudiantes con
TDAH: Un estudio de revisión. ReiDoCrea, 5, 329-340.

Resumen: Los niños con trastorno por déficit de atención con hiperactividad (TDAH) frecuentemente presentan problemas de regulación emocional e interacciones sociales, que derivan en un rechazo y consecuente aislamiento social. El objetivo del presente estudio es realizar una revisión sobre la eficacia de diversas intervenciones de tipo psicosocial, farmacológica o una combinación de ambas, con la finalidad de analizar cómo dichas intervenciones influyen en el comportamiento social de los niños con TDAH. Los resultados obtenidos evidencian que se obtiene una mejora significativa en la adaptación social y emocional de los niños cuando se realiza entrenamiento a padres y profesores. A pesar de que no se obtienen resultados concluyentes en cuanto a conocer si la intervención psicosocial aumenta los beneficios del tratamiento farmacológico, los resultados de esta revisión ponen de manifiesto la importancia de trabajar la competencia social en los niños con TDAH.

Palabras clave: Intervención psicosocial | Trastorno por Déficit de Atención con Hiperactividad (TDAH)

Social competence intervention in students with ADHD: A review study

Abstract: Children with the attention deficit hyperactivity disorder (ADHD) are often accompanied by low competition and social functioning, resulting in rejection and consequent social isolation. The aim of this study is to conduct a review to study the effectiveness of various psychosocial interventions, pharmacological or a combination of both, in order to analyze how these interventions influence the social behavior of children with ADHD. The results show a significant improvement in social and emotional adjustment of children when training parents and teachers. Although no conclusive results are obtained in terms of knowing whether psychosocial intervention enhances the benefits of pharmacotherapy, the results of this review highlight the importance of training social competence in children with ADHD.

Keywords: Attention Deficit Hyperactivity Disorder (ADHD) | Psychosocial Intervention

\section{Introducción}

Las dificultades de atención e hiperactividad fueron descritas por primera vez en 1845 por el psiquiatra Heinrich Hoffmann en el cuento Felipe Nervioso, uno de los diez cuentos que recogía en su obra Pedro Melenas acerca de los problemas psiquiátricos presentes en la infancia y la adolescencia. Posteriormente, George Still describió en 1902 la sintomatología de un grupo de niños que hablan en exceso, que parecen no escuchar, que interrumpen las conversaciones, no respetan las normas sociales, entorpecen las actividades o juegos en grupo; características que se equipararían a lo que actualmente se conoce como Trastorno por Déficit de Atención con Hiperactividad (en adelante TDAH) de presentación clínica inatento, hiperactivo-impulsivo y combinada.

Según el Manual Diagnóstico y Estadístico de los Trastornos Mentales (DSM-5), el TDAH es actualmente conceptualizado como un trastorno del neurodesarrollo caracterizado por un patrón persistente de inatención y/o hiperactividad-impulsividad que interfiere con el funcionamiento o desarrollo de la persona (Asociación Americana de Psiquiatría, 2013). Las investigaciones más recientes confirman que el TDAH es un trastorno neurobiológico, con un fuerte componente genético, en el que los niveles de dopamina se ven afectados, y cuyo curso evolutivo y su pronóstico está influido por factores ambientales (Del Campo, Chamberlain, Sahakian y Robbins, 2011). Además de los síntomas de inatención, impulsividad e hiperactividad, un niño con TDAH 
también sufre un deterioro considerable en otras áreas funcionales, que le llevan a experimentar dificultades interpersonales y de baja competencia social (García, Presentación, Siegenthaler y Miranda, 2006). El patrón interactivo alterado de estos niños provoca el rechazo por parte de los compañeros, con independencia de que presenten o no conductas agresivas. Los indicadores sociométricos, de hecho, evidencian que los niños con TDAH son rechazados por sus compañeros entre un 50 y un 75\% (García et al., 2006; Pardos, Fernández y Fernández, 2009). Investigaciones, como la de García et al. (2006) han obtenido que las razones del rechazo de estos alumnos son debidas en primer lugar a su comportamiento disruptivo y molesto; en segundo lugar a su conducta antisocial; y, en tercer lugar a su agresividad física. Es evidente que este rechazo puede derivar en un aislamiento y la consecuente repercusión del mismo sobre la autoestima del niño.

Según el DSM-IV-TR (2000), la prevalencia del trastorno se sitúa entre el 3 y el $7 \%$ en niños en edad escolar, lo que supone que en un aula podemos encontrar a uno o dos alumnos con TDAH. En el ámbito educativo, las intervenciones con estos estudiantes se centran fundamentalmente en el aspecto académico y puramente comportamental, con el objetivo de mejorar su proceso de enseñanza/aprendizaje y disminuir las conductas disruptivas en el aula. Sin embargo, el aspecto de relación social con los compañeros no suele trabajarse lo suficiente, lo que nos ha motivado a llevar a cabo el presente artículo de revisión con el fin de recalcar la importancia de intervenir en aspectos como la socialización del estudiante, su relación con los compañeros y la afectividad. Del mismo modo, pretendemos sensibilizar a toda la comunidad educativa, mostrar la importancia de la formación del profesorado para trabajar en el aula con una metodología que favorezca las relaciones (aprendizaje cooperativo, por parejas, alumno ayudante/tutor), y sobre todo recalcar las limitaciones que el alumnado con TDAH tiene a nivel de competencia social, limitaciones que pueden verse reducidas con una adecuada intervención.

Son numerosas las investigaciones que relacionan la incidencia del TDAH con dificultades en competencia social. Miranda y Uribe (2001) realizaron una revisión de investigaciones acerca de las interacciones sociales de los niños con TDAH, y destacaron que los fallos en las funciones ejecutivas de éstos afectan de manera considerable a los intercambios comunicativos, a la rigidez en el empleo de estrategias sociales y a la regulación o tratamiento en las expresiones de las emociones. En la misma vertiente, Lora y Moreno (2008) analizaron una muestra de 150 estudiantes de entre 10 y 16 años, y demostraron que los que presentaban sintomatología hiperactiva diferían del grupo de desarrollo típico en casi todas las variables de socialización, a excepción de la variable ansiedad y miedo. Estos autores concluyeron que el negativismo y el retraimiento social acentuaban la sintomatología hiperactiva, mientras que la variable autocontrol, era el principal factor implicado en la disminución de la sintomatología hiperactiva. En otro estudio, Clark Prior y Kinsella (2002) decidieron valorar las diferencias existentes entre adolescentes con TDAH, adolescentes con problemas de conducta y adolescentes de desarrollo típico. Los resultados mostraron que los adolescentes con TDAH presentaban una competencia social tan pobre como el grupo de adolescentes con problemas de conducta pero en cuanto a habilidades de comunicación, los adolescentes con TDAH eran quienes presentaban habilidades más pobres.

La investigación acerca de la eficacia de las intervenciones en relación con la socialización ha sido objeto de estudio de profesionales como Jarque (2012), quien llevó a cabo un análisis sobre las diferentes modalidades de intervención en TDAH en los últimos 25 años. En este estudio, Jarque (2012) evidencia que las intervenciones conductuales, cognitivo-conductuales e instruccionales son eficaces en la reducción de conductas disruptivas y en la mejora del rendimiento académico, mientras que el 
entrenamiento en competencia social mejora el funcionamiento de los niños con conductas antisociales (Webster-Stratton, Reid y Hammond, 2001). De la Peña et al. (2009) realizaron un estudio en el que concluyeron que una intervención adecuada es aquella que aúna medidas farmacológicas con medidas psicosociales, entre las que se encuentran el entrenamiento en habilidades sociales. Por otra parte, trabajos como el de Barkley (2002) plantean que la intervención con los padres también proporciona mejores resultados en el funcionamiento social de los niños.

Como hemos podido observar, numerosos estudios han demostrado las dificultades interpersonales y la baja competencia social que presentan los niños con TDAH. La adaptación de estos niños a su entorno no resulta posible sin un adecuado desarrollo de las habilidades sociales. Este hecho ha propiciado que en muchas investigaciones se incluyan escalas de habilidades sociales, cuyas puntuaciones facilitan tanto el diagnóstico como el tratamiento. Este es el caso del estudio llevado a cabo por Fernández et al. (2011), en el que se analizó de manera retrospectiva a 170 pacientes con diagnóstico de TDAH, y se demostró que las habilidades sociales medidas a través del Sistema de Evaluación de la Conducta de Niños y Adolescentes (BASC, 2011), se relacionan, en opinión de los padres, con la intensidad sintomática del TDAH y con la presencia de comorbilidad conductual. No se obtuvieron datos estadísticamente significativos respecto a éstas u otras variables en el caso de que fueran los profesores los informadores. Lo que sí se demostró es que puntuaciones elevadas de "hiperactividad" mostraron de manera contundente una peor competencia social.

La actual Ley Orgánica de Mejora de la Calidad Educativa (LOMCE, 2013) ha supuesto, por primera vez a nivel estatal, la inclusión del TDAH como una necesidad específica de apoyo educativo (art. 71.2), además de que se ha añadido un artículo nuevo en el que establece el derecho a la identificación y valoración de sus necesidades educativas y la consiguiente intervención lo más tempranamente posible (art. 79bis). Aunque en determinadas comunidades autónomas ya se tenía en consideración el TDAH como necesidad específica, el hecho de que se haya reconocido a nivel estatal denota, por un lado, la alta prevalencia de casos, y por otro, la importancia de una rápida detección e intervención con el fin de atender a las necesidades de estos alumnos.

Vemos, por tanto, que numerosos estudios señalan las dificultades sociales que los niños y adolescentes con TDAH presentan, y cómo el hecho de incidir en la mejora de las habilidades sociales puede suponer una intervención efectiva. El objetivo del presente trabajo es realizar una revisión de artículos que nos permita analizar qué intervenciones resultan más efectivas para la mejora de las competencias sociales de las personas con TDAH, con la finalidad de reflexionar acerca de las implicaciones educativas que dichos resultados pueden ofrecernos de cara a la práctica educativa.

\section{Método}

\section{Revisión bibliográfica}

Para el desarrollo de la presente revisión se han utilizado las siguientes bases de datos: PsycINFO -base de datos de la Asociación Americana de Psicología-; Psyke catálogo de artículos sobre Psicología-; ERIC -Educational Resources Information Center-; OvidSP -plataforma de búsqueda de bases de datos-; ProQuest -base de datos que publica archivos de fuentes diversas-; Wiley Online Library -base que permite consultar revistas de diversas disciplinas-; la biblioteca virtual de la Universitat Oberta de Catalunya; el buscador Trobes Plus de la Universidad de Valencia; y el 
Google Scholar, donde además de buscar artículos a través de las palabras claves, se utilizó el recurso de "citado por" y "artículos relacionados", facilitando así la búsqueda de otros textos. Las palabras clave utilizadas han sido: Trastorno por Déficit de Atención con Hiperactividad (TDAH); relaciones sociales; socialización; habilidades sociales; intervención psicosocial; estado sociométrico; conducta disruptiva; tratamiento multimodal; tratamiento farmacológico; metilfenidato; y, revisión bibliográfica. La búsqueda de todas estas palabras se realizó tanto en castellano como en catalán e inglés, con el fin de obtener el mayor número de resultados posibles.

\section{Procedimiento}

Para la realización del presente artículo de revisión, en principio se estableció un período de cinco años desde la fecha actual, pero posteriormente se decidió ampliar el período desde 1997, ya que consideramos interesante el hecho de comparar las primeras intervenciones que se llevaban a cabo con las intervenciones que se están efectuando en estos últimos años. De este modo se puede comprobar si el procedimiento de intervención ha cambiado de manera considerable, o si con intervenciones similares se obtienen diferentes resultados en función de cuándo fueron aplicadas.

\section{Criterio de inclusión}

El criterio de inclusión fundamental fue que fueran trabajos empíricos, en los que se llevara a cabo una intervención y por tanto: a) se describiera la muestra con la que iban a trabajar (número de participantes, edad, diagnóstico de los mismos); b) se especificaran los instrumentos que iban a utilizar para llevar a cabo la intervención (pruebas diagnósticas, recursos materiales y humanos); c) se describiera la intervención concreta que se iba a llevar a cabo con la muestra y los pasos que se iban a seguir para la realización de la misma; d) y que se expusieran los resultados obtenidos tras dicha intervención.

Este criterio supuso que quedaran descartados todos aquellos artículos de reflexión acerca de TDAH y su influencia en la competencia social. Del mismo modo, quedaron descartados todos aquellos artículos descriptivos que analizaban el estado de la cuestión y aquellos artículos cuyo objetivo era la revisión de artículos anteriores. De los treinta y cinco artículos que se obtuvieron inicialmente diez cumplían los criterios de inclusión establecidos, siendo estos representativos de las intervenciones en el área de competencia social. Se han seleccionado estos diez trabajos para mostrar la situación actual y la evolución del tema. En la Tabla 1 se incluye un resumen de los mismos.

\begin{tabular}{|c|c|c|c|c|}
\hline Referencia & $\mathbf{N}$ & Variables dependientes y medida & Contenidos de la intervención & Resultados \\
\hline $\begin{array}{l}\text { Abikoff et al. } \\
(2004)\end{array}$ & $\begin{array}{l}103 \text { niños: } 7-9 \\
\text { años con TDAH. }\end{array}$ & $\begin{array}{l}\text { Función social y comportamiento } \\
\text { de compañeros y profes. Medidas a } \\
\text { los } 6,12,18 \text { y } 24 \text { meses. } \\
\text { Instrumentos: SSRS, Taxonomy of } \\
\text { Problem Situation, Código de } \\
\text { Observación de Interacción Social. }\end{array}$ & $\begin{array}{l}3 \text { grupos: sólo metilfenidato; } \\
\text { metilfenidato + tratamiento psicosocial } \\
\text { multimodal (incluye HHSS); } \\
\text { metilfenidato + atención de control. } \\
\text { Duración: } 2 \text { años }\end{array}$ & $\begin{array}{l}\text { El metilfenidato + } \\
\text { tratamiento multimodal no } \\
\text { genera mejores resultados } \\
\text { con respecto a los otros dos } \\
\text { grupos. }\end{array}$ \\
\hline \multirow[t]{2}{*}{$\begin{array}{l}\text { Beh-Pajooh, } \\
\text { Fatemi, Bonab, } \\
\text { Alizadeh y } \\
\text { Hemmati (2012) }\end{array}$} & $\begin{array}{l}20 \text { niños: } 7-11 \\
\text { años con TDAH. }\end{array}$ & $\begin{array}{l}\text { Habilidades sociales: pretest - } \\
\text { postest - seguimiento. Grupo } \\
\text { control y experimental. } \\
\text { Instrumentos: lista verificación } \\
\text { TDAH del DSM-IV, Matrices } \\
\text { progresivas de Raven, Escala } \\
\text { SSRS. }\end{array}$ & $\begin{array}{l}\text { Grupo experimental: programa de } \\
\text { entrenamiento autocontrol. } \\
4 \text { Fases: enseñanza de HHSS y } \\
\text { contingencia; economía de fichas } \\
\text { maestros; economía de fichas } \\
\text { alumnos; recompensa. } \\
\text { Duración: } 1 \text { mes. }\end{array}$ & $\begin{array}{l}\text { El procedimiento de } \\
\text { formación en autocontrol fue } \\
\text { significativamente eficaz en } \\
\text { la mejora de la competencia } \\
\text { social. }\end{array}$ \\
\hline & & $\begin{array}{l}\text { Comportamiento social adecuado, } \\
\text { conducta disruptiva en la }\end{array}$ & $\begin{array}{l}\text { Tratamiento multimodal: } \\
\text { intervenciones conductuales; }\end{array}$ & $\begin{array}{l}\text { Reducción de conducta } \\
\text { social inapropiada y del }\end{array}$ \\
\hline
\end{tabular}




\begin{tabular}{|c|c|c|c|c|}
\hline $\begin{array}{l}\text { Northup } \\
\text { Reitman y Back } \\
(2009)\end{array}$ & $\begin{array}{l}22 \text { niños: } 4-6 \\
\text { años con TDAH } \\
\text { tipo combinado. } \\
\text { Padres. }\end{array}$ & $\begin{array}{l}\text { intervención temprana. } \\
\text { Instrumentos: Manual for the } \\
\text { ASEBA School-Age Form \& } \\
\text { Profiles, DSM-IV, CBRS, IPE, } \\
\text { SDSERS, ECBI. }\end{array}$ & $\begin{array}{l}\text { medicación+tratamiento conductual; } \\
\text { entrenamiento combinado HHSS y } \\
\text { deportivo; programa de entrenamiento } \\
\text { para padres. Duración: } 6 \text { semanas. }\end{array}$ & $\begin{array}{l}\text { comportamiento disruptivo. } \\
\text { Efectividad de la } \\
\text { intervención temprana. }\end{array}$ \\
\hline $\begin{array}{l}\text { O' Callaghan, } \\
\text { Reitman, } \\
\text { Northup, Hupp } \\
\text { y Murphy } \\
\text { (2003) }\end{array}$ & $\begin{array}{l}4 \text { niños con } \\
\text { TDAH. }\end{array}$ & $\begin{array}{l}\text { Conducta prosocial, conducta } \\
\text { atenta y comportamiento } \\
\text { deportivo.Instrumentos: DSM-IV, } \\
\text { CPRS-R, } \\
\text { SSRS. }\end{array}$ & $\begin{array}{l}\text { Entrenamiento: modelización } \\
\text { adecuada de HHSS, } \\
\text { retroalimentación, economía de fichas } \\
\text { para favorecer la adquisición de HHSS } \\
\text { en contexto deportivo. Programa de } \\
\text { generalización. Duración: } 6 \text { semanas. }\end{array}$ & $\begin{array}{l}\text { Conducta prosocial, atenta y } \\
\text { comportamiento deportivo se } \\
\text { ven incrementados tras la } \\
\text { intervención. El programa de } \\
\text { generalización aumentó la } \\
\text { transferencia al entorno } \\
\text { deportivo. }\end{array}$ \\
\hline $\begin{array}{l}\text { Pfiffner y } \\
\text { McBurnett } \\
\text { (1997) }\end{array}$ & $\begin{array}{l}27 \text { niños: } 25 \text { con } \\
\text { TDAH y } 2 \text { con } \\
\text { Déficit de } \\
\text { Atención } \\
\text { Indiferenciado } \\
\text { Padres. }\end{array}$ & $\begin{array}{l}\text { Conocimiento de habilidades, } \\
\text { conducta social y conducta } \\
\text { disruptiva. Transferencia al entorno } \\
\text { familiar y escolar. Pretest, postest } \\
\text { y seguimiento. } \\
\text { Instrumentos: SSRS, Escala UCI, } \\
\text { CLAM, SNAP-R, CBCL, TRF, } \\
\text { cuestionario de satisfacción. }\end{array}$ & $\begin{array}{l}3 \text { grupos: programa breve de HHSS } \\
\text { con generalización mediada de } \\
\text { padres; programa breve de HHSS sólo } \\
\text { para los niños; y grupo control. } \\
\text { Duración: } 8 \text { semanas. }\end{array}$ & $\begin{array}{l}\text { Mejora de habilidades } \\
\text { sociales y conducta } \\
\text { disruptiva en los dos grupos } \\
\text { de tratamiento. Mayor } \\
\text { transferencia al entorno } \\
\text { escolar del grupo de } \\
\text { entrenamiento a padres. }\end{array}$ \\
\hline $\begin{array}{l}\text { Presentación } \\
\text { Siegenthaler, } \\
\text { Jara y Miranda } \\
(2010)\end{array}$ & $\begin{array}{l}27 \text { niños: } 7-10 \\
\text { años con TDAH. } \\
\text { Padres. } \\
\text { Profesores. }\end{array}$ & $\begin{array}{l}\text { Adaptación académica, emocional } \\
\text { y social: pretest - postest - } \\
\text { seguimiento. } \\
\text { Instrumentos: Cuestionario TDAH } \\
\text { del DSM-IV-TR, WISC-R, EPC, } \\
\text { IPE, Cuestionario hiperactividad y } \\
\text { agresividad IOWA, sociometría. }\end{array}$ & $\begin{array}{l}3 \text { programas: intervención para niños, } \\
\text { para padres y para profesores: } \\
\text { técnicas cognitivo conductuales, } \\
\text { técnicas de modificación de conducta, } \\
\text { modificaciones instruccionales. } \\
\text { Duración: niños } 16 \text { sesiones; adultos y } \\
\text { padres } 10 \text { sesiones. }\end{array}$ & $\begin{array}{l}\text { Adaptación emocional: } \\
\text { mejora de timidez, y } \\
\text { problemas de ansiedad. } \\
\text { Adaptación social: mejora de } \\
\text { la conducta antisocial y de la } \\
\text { agresividad. }\end{array}$ \\
\hline $\begin{array}{l}\text { Reitman, } \\
\text { O'Callaghan y } \\
\text { Mitchell (2005) }\end{array}$ & $\begin{array}{l}\text { Niños con TDAH. } \\
\text { Padres. }\end{array}$ & $\begin{array}{l}\text { Habilidades sociales, } \\
\text { comportamiento prosocial y } \\
\text { antisocial. Instrumentos: SSRS, } \\
\text { CBRS. }\end{array}$ & $\begin{array}{l}\text { Niños: sesiones individuales de } \\
\text { coaching, modelado. } \\
\text { Padres: Programa de entrenamiento } \\
\text { en HHSS para promover en sus hijos } \\
\text { la conducta social adecuada y las } \\
\text { HHSS. } \\
\text { Duración: } 12 \text { semanas. }\end{array}$ & $\begin{array}{l}\text { Mejora de la conducta } \\
\text { prosocial, reducción del } \\
\text { comportamiento agresivo, } \\
\text { integración en deporte de } \\
\text { equipo y formación de } \\
\text { nuevas amistades. }\end{array}$ \\
\hline $\begin{array}{l}\text { Storebo, Gluud, } \\
\text { Winkel y } \\
\text { Simonsen } \\
(2012)\end{array}$ & $\begin{array}{l}56 \text { niños: 8-12 } \\
\text { años con TDAH. } \\
\text { Padres. }\end{array}$ & $\begin{array}{l}\text { Competencias sociales y } \\
\text { emocionales. } \\
\text { Grupo control y experimental. } \\
\text { Instrumentos: KSADS, SCQ, } \\
\text { ASRS, WISC-III, CBRS. }\end{array}$ & $\begin{array}{l}\text { Grupo control: medicación. } \\
\text { Grupo experimental: medicación + } \\
\text { entrenamiento en HHSS + } \\
\text { entrenamiento a padres. } \\
\text { Duración: niños } 8 \text { sesiones y padres } 3 \\
\text { sesiones. }\end{array}$ & $\begin{array}{l}\text { El entrenamiento en HHSS y } \\
\text { formación a padres no } \\
\text { mostró mayores beneficios } \\
\text { en comparación con el } \\
\text { tratamiento estándar. }\end{array}$ \\
\hline $\begin{array}{l}\text { Wilkes Cordier, } \\
\text { Bundy, Docking } \\
\text { y Munro (2011) }\end{array}$ & $\begin{array}{l}30 \text { niños: } 5-11 \\
\text { años. } 15 \text { con } \\
\text { TDAH y } 15 \text { sin } \\
\text { TDAH. } \\
\text { Padres. }\end{array}$ & $\begin{array}{l}\text { Mejora del juego social, de las } \\
\text { HHSS y empatía interpersonal. } \\
\text { Pretest - postest. } \\
\text { Instrumentos: CPRS-3, CBCL, Test } \\
\text { of Playfulness. }\end{array}$ & $\begin{array}{l}\text { Niños: uso del juego, } \\
\text { retroalimentación, modelado de } \\
\text { terapeuta, modelado de pares, auto- } \\
\text { modelado. Padres: formación en } \\
\text { estrategias de intervención en otros } \\
\text { contextos. Duración: } 7 \text { semanas. }\end{array}$ & $\begin{array}{l}\text { Tanto los alumnos con } \\
\text { TDAH como los alumnos sin } \\
\text { TDAH mejoran el juego } \\
\text { social y las habilidades } \\
\text { sociales. }\end{array}$ \\
\hline
\end{tabular}

Notas. SSRS=Escala de Clasificación de Habilidades Sociales; WISC=Escala Wechsler de Inteligencia; EPC=Escala de Problemas de Conducta para padres; IPE=Inventario de Problemas en la Escuela; CBRS=Escala de Conducta Conners Revisada; SDSERS=Escala de Clasificación de los Efectos Secundarios de Estimulantes; ECBI=Inventario de Comportamiento Eyberg para niños; CPRS-R=Escala de clasificación Conners para padres; UCI=Escala de Habilidades Sociales de la Universidad de California, Irvine; CLAM=Escala de Conners, Loney y Milich; SNAP-R=Escala de Swanson, Nolan y Pelham; CBCL=Lista de verificación de la conducta infantil; TRF=Formulario de valoración del maestro; KSADS=Programa para trastornos afectivos y esquizofrenia para niños en edad escolar; SCQ=Cuestionario de comunicación social; ASRS=Escala de autoinforme para detección de síntomas de TDAH; $\mathrm{BASC}=$ Escala de evaluación de la conducta del niño; SCRS=Escala de autocontrol; SS=Encuesta de satisfacción.

\section{Resultados}

Considerando las intervenciones realizadas en los diez artículos seleccionados, a continuación se presenta un análisis de los diferentes estudios, los cuales han sido 
agrupados en cuatro categorías. Estas cuatro categorías son: modalidad de tratamiento (intervención conductual versus combinación de tratamiento farmacológico con intervención psicosocial), participantes en la intervención (niños, padres y profesorado), etapa de intervención (importancia de la intervención temprana) y generalización de los resultados tras la intervención.

\section{Modalidad de tratamiento}

Estudios como el de Beh-Pajooh et al. (2012) demuestran la eficacia de enseñar técnicas de autocontrol a alumnos con TDAH. Para estudiar la eficacia de este tipo de intervención, los participantes con TDAH se dividieron en un grupo experimental y un grupo control. Los resultados evidenciaron diferencias significativas entre ambos grupos, de manera que aquellos alumnos a los que se les enseñó técnicas de autocontrol y habilidades sociales apropiadas, obtuvieron una mejoría en cuanto a su capacidad de autocontrol, disminución de los problemas de comunicación, comportamientos problemáticos y habilidades sociales.

Otros estudios han analizado la efectividad de combinar la intervención psicosocial con el tratamiento farmacológico para la mejora del funcionamiento social de los niños con TDAH. Este es el caso de estudios de seguimiento a largo plazo, como el Tratamiento Multimodal MTA (MTA Cooperative Group, 2004), el cual ha evidenciado una mayor efectividad del tratamiento farmacológico, ya fuese sólo o combinado con intervención no específica. Abikoff et al. (2004), por el contrario, hipotetizaron que el metilfenidato en combinación con una intervención psicosocial multimodal intensiva mejoraría de manera significativa el funcionamiento social de los niños con TDAH, en comparación con un exclusivo tratamiento farmacológico. Para ello, se formaron tres grupos: metilfenidato+intervención psicosocial, sólo metilfenidato y metilfenidato+atención de control. Los resultados muestran que los tres grupos mejoraron en la calificación de las habilidades sociales y los problemas de conducta reportados por padres y profesores, pero sin llegar a obtener diferencias significativas entre ellos, por lo que no pudo demostrarse la hipótesis planteada por los autores. En el único aspecto en que se encontraron diferencias es que el grupo metilfenidato+intervención psicosocial recibió más reacciones positivas por parte de compañeros y profesores. En el estudio de Storebo et al. (2012) establecieron un grupo control, que recibía el tratamiento estándar (medicación), y un grupo experimental que, además de recibir la medicación, recibía entrenamiento en competencia social y formación a padres. Los resultados evidenciaron que no hay diferencias significativas en las competencias sociales y emocionales entre los niños que formaron el grupo experimental y los que recibieron el tratamiento estándar.

\section{Participantes en la intervención}

Diversos trabajos no sólo se han centrado en la intervención en niños, sino que han ampliado su intervención a padres y profesorado.

En esta línea, Sim et al. (2006), además de realizar una intervención con niños, instruyéndolos en conductas prosociales y manejo de la ira, realizaron una intervención con padres para que aprendieran cómo proporcionar oportunidades para que los niños practiquen habilidades sociales. Del mismo modo también intervinieron con los maestros para trabajar el reforzamiento de conductas específicas de estos niños. Los resultados evidenciaron una diferencia significativa pre y post-tratamiento en cuanto a agresión, externalización del comportamiento y autocontrol. 
En otro estudio, Presentación et al. (2010) plantearon una intervención en técnicas cognitivo-conductuales, técnicas autoinstruccionales, solución de problemas, control de la ira y habilidades sociales, añadiendo además la participación de padres y profesores. A los padres se les formó en técnicas de modificación de conducta, apoyo al aprendizaje escolar y afrontamiento de situaciones complejas de crianza. A los profesores también se les formó en técnicas de modificación de conducta, en cómo motivar al alumnado al utilizar las técnicas autoinstruccionales y en cómo realizar adaptaciones físicas, organizativas e instruccionales. Los resultados reflejan una mejora significativa en las variables timidez y ansiedad valorada por padres y profesores, y una mejora de la variable retraimiento informada por los padres, pero no por el profesorado. En cuanto a la adaptación social, se observa una mejoría significativa en la conducta antisocial, valorada tanto por padres como por profesores, así como una mejoría en agresividad y adaptación escolar, mejorías que se mantuvieron incluso en la fase de seguimiento, un año después de la intervención.

\section{Etapa de intervención}

Algunos trabajos, como el de Northup et al. (2009), han abordado la cuestión sobre la importancia de la intervención temprana en niños con TDAH. Estos autores aplicaron una intervención multimodal a preescolares entre 4 y 6 años durante 6 semanas. Los resultados evidenciaron que con una intervención temprana ya pueden conseguirse cambios a nivel de comportamiento social y conducta disruptiva; de ahí la importancia de llevar a cabo una detección e intervención lo más tempranamente posible. Del mismo modo, el programa de intervención en funcionamiento ejecutivo para preescolares llamado The Tools of the Mind (Diamond, Barnett, Thomas y Munro, 2007) ha evidenciado que un entrenamiento temprano mejora el rendimiento de los niños en tareas escolares. Programas de intervención con padres en edades tempranas, como el New Forest Parenting Package (Sonuga-Barke, Thompson, Abikoff, Klein, y Brotman, 2006) y el Triple P Positive, Parenting, Program (De Graaf, Speetjens, Smit, de Wolff, y Tavecchio, 2008) también han puesto de manifiesto la importancia de la intervención con los padres para mejorar los problemas de conducta. El programa Incredible Years (Webster-Stratton, 2011) además de un entrenamiento con niños de 2 a 8 años, propone un entrenamiento tanto a padres como a profesores para promover la competencia socioemocional de los niños con TDAH.

\section{Generalización de los resultados tras la intervención}

El hecho de que los resultados conseguidos tras la intervención puedan mantenerse y generalizarse a otros contextos, fuera del entorno terapéutico controlado, también ha sido objeto de diversos estudios, obteniendo resultados satisfactorios.

Wilkes et al. (2011) utilizaron el contexto de juego entre iguales como base para mejorar las habilidades sociales de los niños con TDAH, basándose en estudios que indican un desarrollo inapropiado del juego y falta de respuesta empática en estos niños (Cordier, Bundy, Hocking y Einfeld, 2010). Se establecieron parejas de juego entre un niño sin TDAH y otro niño con este trastorno, y se les aplicaron técnicas de modelado, auto-modelado y retroalimentación. Los resultados mostraron que la intervención dio lugar a mejoras en el juego social, las habilidades sociales y la empatía interpersonal de todos los niños, tuviesen o no el trastorno. En un estudio similar, O'Callaghan et al. (2003) también obtuvieron que tras una intervención directa con los niños en habilidades sociales, retroalimentación, refuerzo y economía de fichas, la transferencia al entorno de juego pasó a ser del $7 \%$ al $56 \%$ en conducta atenta y del $10 \%$ al $40 \%$ en comportamiento deportivo. 
Otros estudios han tratado de entrenar a los padres con la finalidad de que estos apoyaran la transferencia de las habilidades sociales de sus hijos al contexto del día a día. Para analizar la efectividad de este tipo de intervenciones, Pfiffner y McBurnett (1997) dividieron la muestra en tres grupos: un grupo control; un grupo al que sólo se le aplicaba el programa de habilidades sociales a los niños; y un tercer grupo en el que se aplicaba dicho programa a los niños y se entrenaba a los padres para la generalización de las habilidades. Los resultados mostraron que los niños de ambos grupos experimentales mejoraron sus habilidades sociales y disminuyeron su conducta disruptiva. En cuanto a la transferencia de habilidades al entorno hogar, no se obtuvieron diferencias significativas. Sin embargo, sí se encontró que el grupo de entrenamiento a padres supuso mayor transferencia al entorno escolar. En la misma línea, Reitman et al. (2005) entrenaron a los padres en competencia social en entornos deportivos. El hecho de instruir a los padres en principios básicos de análisis y manejo de la conducta, como el uso del refuerzo, tiempo fuera e instrucciones, dio como resultado que los niños redujeran su conducta social inapropiada y su comportamiento agresivo, además de que favoreció su integración en un deporte de equipo y establecieron nuevas amistades.

\section{Discusión}

Gran cantidad de estudios señalan que los niños con TDAH presentan una pobre competencia social y débiles relaciones interpersonales (Anhalt, McNeil y Bahl, 1998; Kotkin, 1998), lo que conlleva que un porcentaje elevado de ellos experimenten rechazo por parte de sus compañeros y relaciones problemáticas con los adultos (Stahl, 2007). Este rechazo se asocia a efectos adversos inmediatos y a consecuencias a largo plazo como son las dificultades de adaptación social (Kupersmidt y Dodge, 2004).

En esta revisión los diferentes estudios analizados tienen como principal objetivo valorar como el trabajo en competencia social puede conllevar mejoras en el funcionamiento social de los niños con diagnóstico de TDAH. Los resultados obtenidos en la mayoría de estudios muestran que tras una intervención psicosocial, se produce una mejora significativa de la adaptación tanto social como emocional de estos niños. Estos resultados son respaldados por numerosas investigaciones que consideran la aplicabilidad de programas de entrenamiento en competencia social como una intervención de éxito tanto con niños con TDAH rechazados por sus compañeros (Micou, 2003), como con niños que presenten conductas agresivas o problemas de aislamiento social. (Kotkin, 1998; Pelham et al., 2005)

En referencia a la relación con iguales, algunas investigaciones corroboran que la falta de atención, la impulsividad y un comportamiento social inapropiado generan relaciones negativas con los iguales (Johnson y Rosen, 2000), hasta el punto de ser rechazos por parte de los compañeros (Stahl, 2007), lo que como consecuencia conlleva problemas graves en el juego, así como dificultad para hacer y mantener amigos. Los resultados de esta revisión parecen indicar que el hecho de trabajar las habilidades sociales, el comportamiento prosocial y la mejora del juego social, supone que el niño puede llegar a reducir su comportamiento agresivo, ser capaz de establecer amistades y mejorar su juego social y su integración en un deporte de equipo. Es importante destacar que en el caso del estudio de Wilkes et al. (2011) dicha intervención mejoró tanto el juego social como las habilidades sociales de todos los niños, tuviesen o no el trastorno. Considerando este resultado, por tanto, es evidente que para conseguir la inclusión del alumno con TDAH en el contexto de iguales, la intervención no sólo ha de centrarse en este niño en concreto, sino que se debe trabajar con todos los niños que forman parte de su entorno. 
Del mismo modo que se debe realizar una intervención con los iguales, también resulta positivo que se realice un entrenamiento tanto a padres como a profesores. Los resultados encontrados parecen indicar que la formación tiene efectos positivos en la adaptación social y emocional de los niños y en la disminución de la conducta agresiva (Kotkin, 1998; Pelham et al., 2005). Es importante que la intervención con padres y profesores se realice de manera coordinada, estableciendo los mismos objetivos de trabajo, ya que ello va a favorecer a que el cambio se produzca de manera más rápida y que se mantenga a lo largo del tiempo. Es imprescindible transmitir a padres y profesores la importancia de su formación e intervención para poder conseguir una máxima implicación. A pesar de los resultados tan favorables que se obtienen en los estudios analizados de Sim et al. (2006) y de Presentación et al. (2010), cabe señalar que en ninguno de estos trabajos se estableció un grupo control, por lo que no puede determinarse si la efectividad de este tipo de tratamiento es significativa. Además, también sería necesario llevar a cabo estudios a más largo plazo que puedan corroborar la eficacia de las intervenciones realizadas a corto plazo.

Otros estudios de análisis entrenaron a los padres con el objetivo de generalizar los resultados obtenidos a otros contextos. En los estudios analizados al respecto, la participación de los padres dio lugar a una mayor transferencia de los resultados al contexto de juego (O'Callaghan et al., 2003) y al contexto escolar, aunque para el contexto del hogar no se obtuvieron resultados concluyentes (Pfiffner y McBurnett, 1997). Esto apoya igualmente la importancia de la implicación y la participación activa de los padres en el tratamiento de su hijo o hija. Asimismo, debemos destacar que los resultados de algunos de los trabajos demuestran que tanto el contexto del juego, como el del deporte, son contextos facilitadores para el trabajo y la transferencia del funcionamiento social (Reitman et al. 2005; Wilkes et al., 2011). Esto implica lo favorable que puede resultar hacer partícipe al alumno con TDAH en una actividad deportiva para la mejora de sus competencias sociales. En esta línea, estudios como los de Hupp, Reitman, Northup, O'Callaghan y LeBlanc (2002) destacan la importancia de aprovechar el contexto deportivo como medio de promoción del desarrollo social.

Respecto a la importancia de la intervención temprana, la estructura curricular de la etapa de Educación Infantil se considera como un contexto facilitador para trabajar las habilidades sociales a través del juego. Tal y como se ha señalado previamente, la nueva ley orgánica de educación (LOMCE, 2013) destaca la importancia de una intervención lo más temprana posible. No obstante, la investigación sobre la relación entre competencia social y los niños en edad preescolar con riesgo de presentar TDAH es más bien escasa y todavía está en desarrollo (Kern et al., 2007). De hecho, en esta revisión, considerando los criterios de inclusión, solamente se ha seleccionado un artículo al respecto, el cual evidencia que una intervención multimodal en edades tempranas conlleva mejoras significativas a nivel de comportamiento social y conducta disruptiva (Northup et al., 2009).

En cuanto a los estudios analizados en los que además de una intervención psicosocial, los sujetos recibieron tratamiento farmacológico, no parece que la intervención psicosocial suponga un plus en los beneficios de la medicación a nivel de mejora de las habilidades y funcionamiento social (Abikoff et al., 2004; Storebo et al., 2012). No obstante, existen publicaciones muy contradictorias al respecto, ya que mientras algunas de ellas plantean que la medicación no facilita la adquisición de habilidades sociales básicas (Pelham, Wheeler y Chronis, 1998), otras publicaciones plantean que el tratamiento farmacológico reduce los comportamientos sociales negativos (MTA Cooperative Group, 2004). A la vista de los resultados obtenidos, por tanto, no puede valorarse hasta qué punto los pacientes que reciben tratamiento farmacológico, pueden verse o no beneficiados por un programa de entrenamiento en competencia social. 
Este trabajo presenta una serie de limitaciones, como son: el reducido número de artículos revisados y, el hecho de no contemplar otras variables como pueden ser los problemas de conducta, la autoestima, la ansiedad o la depresión; variables muy estrechamente relacionadas con el TDAH, y que podrían ser objeto de futuras investigaciones.

Pese a estas limitaciones, el presente trabajo puede resultar de gran ayuda para aquellos profesionales que en su día a día trabajan con alumnos con TDAH y no encuentran respuesta a muchas de las reacciones de estos alumnos y al ambiente relacional que se establece entre compañeros. Del mismo modo, puede resultar de ayuda a los padres de estos niños para que puedan ver la importancia de su implicación en el trabajo con sus hijos, además de la generalización que puede conllevar su trabajo a otros contextos. Finalmente, este trabajo también puede servir de estímulo para profesionales de la investigación, ya que todavía queda mucho por investigar, con el objetivo final de mejorar la calidad de vida de las personas con TDAH.

\section{Referencias}

Abikoff, H., Hechtman, L., Klein, R., Gallagher, R., Fleiss, K., Etcovitch, J., ... y Pollack, S. (2004). Social functioning in children with ADHD treated with long-term methylphenidate and multimodal psychosocial treatment. Journal American Academy of Child and Adolescent Psychiatry, 43(7), 820-829.

American Psychiatric Association (2000). Diagnostic and statistical manual of mental disorders (4th ed., text rev.) Washington DC: Author.

American Psychiatric Association. (2013). Diagnostic and Statistical Manual of Mental Disorders (5th. Ed.) Editorial Médica Panamericana.

Anhalt, A., McNeil, C.B., y Bahl, A.B. (1998). The ADHD classroom kit: a whole-classroom approach for managing disruptive behavior. Psychology in the schools, 35(1), 67-79.

Barkley, R. A. (2002). Psychosocial treatments for attention-deficit/hyperactivity disorder in children. Journal of Clinical Psychiatry, 63, 36-43.

Beh-Pajooh, A., Fatemi, S., Bonab, B., Alizadeh, H., y Hemmati, G. (2012). The impact of a self-sontrol training program on enhancement of social skills in students with ADHD. Scientific Research, 3(8), 616-620.

Clark, C.H., Prior, M., y Kinsella, G. (2002). The relationship between executive function abilities, adaptative behaviour, and academic achievement in children with externalising behaviour problems. Journal of Child Psychology \& Psychiatry, 43, 785-796.

Cordier, R., Bundy, A., Hocking, C., y Einfeld, S. (2010). Empathy in the play of children with ADHD. OTJR, Occupation, Participation, and Health, 30(3), 122-132.

De Graaf, I., Speetjens, P., Smit, F., de Wolff, M., y Tavecchio, L. (2008). Effectiveness of the Triple P Positive Parenting Program on behavioral problems in children: a meta-analysis. Behavior Modification, 32, 714-735.

De la Peña, F., Barragán, E., Rohde, L., Patiño L., Zavaleta, P., Ulloa, R., ... y Larraguibel, M. (2009). Algoritmo de Tratamiento Multimodal para Escolares Latinoamericanos con Trastorno por Déficit de Atención con Hiperactividad (TDAH). Salud Mental, 32, 17-29.

Del Campo, N., Chamberlain, S.R., Sahakian, B.J., y Robbins, T.W. (2011). The roles of dopamine and noradrenaline in the pathophysiology and treatment of ADHD. Biological Psychiatry, 69, 145-167.

Diamond, A., Barnett, W.S., Thomas, J., y Munro, S. (2007). Preschool program improves cognitive control. Science (New York, NY), 318(5855), 1387.

Fernández, A., Fernández, D., López, S., García, C., Muñiz, B., Pardos, A., ... y Muñoz, N. (2011). Trastorno por déficit de atención/ hiperactividad y su relación con las habilidades sociales y de liderazgo evaluadas a través de un sistema de evaluación de la conducta de niños y adolescentes (BASC). Actas Esp. Psiquiatr., 39(6), 339-348. 
García, R., Presentación, M.J., Siegenthaler, R. y Miranda, A. (2006). Estado sociométrico de los niños con trastorno por déficit de atención con hiperactividad subtipo combinado. Revista de Neurología, 42(2), 13-18.

Hupp, S.D., Reitman, D., Northup, J., O'Callaghan, P., y LeBlanc, M. (2002). The effects of delayed rewards, tokens, and stimulant medication on sportsmanlike behavior with ADHD-diagnosed children. Behavior Modification, 26(2), 148-162.

Jarque, S. (2012). Eficacia de las intervenciones con niños y adolescentes con Trastorno por Déficit de Atención con Hiperactividad (TDAH). Anuario de Psicología, 42(1), 19-33.

Johnson, R., y Rosen, L. (2000). Sports behavior of ADHD children. Journal of Attention Disorders, 4, 150-160.

Kern, L., DuPaul, G., Volpe, R., Sokol, N., Lutz, G., Arbolino, L., ... y VanBrakle, J.D. (2007). Multisetting assessmentbased intervention for young children at risk for attention deficit hyperactivity disorder. School Psychology Review, 36(2), 237-255.

Kotkin, R. A. (1998). The Irvine Paraprofessional Program: Promising practice for serving students with ADHD. Journal of Learning Disabilities, 31, 556-564.

Kupersmidt, J., y Dodge, K. (2004). Children's peer relations: From development to intervention. Decade of behavior. Washington DC: American Psychological Association.

Ley orgánica 8/2013, de 9 de diciembre, para la mejora de la calidad educativa, BOE núm. 295 § 97858 (2013).

Lora, J.A., y Moreno, I. (2008). Perfil social de los subtipos del Trastorno por Déficit de Atención con Hiperactividad. Apuntes de psicología, 26(2), 317-329.

Micou, L.L. (2003). Social evaluations of children with ADHD participating in peer pairing interventions: Disconfirming behavior versus peer association. Northern Illinois University.

Miranda, A., y Uribe, L.H. (2001). Funciones ejecutivas. Teoría de la mente y competencia social de niños con TDAH. En Miranda, A., Actas del l Congreso Internacional de Déficit de Atención y Dificultades de Aprendizaje (pp. 47-68). Málaga: Aljibe.

MTA Cooperative Group. (2004). National Institute of Mental Health Multimodal Treatment Study of ADHD follow-up: 24month outcomes of treatment strategies for attention-deficit/hyperactivity disorder. Pediatrics, 113(4), 754-761.

Northup, J., Reitman, D., y Back, J. (2009). The star program: A description and analysis of a multifaceted early intervention for young children with a diagnosis of attention deficit hyperactivity disorder. Child \& Family Behavior Therapy, 31(2), 75-93.

O'Callaghan, P., Reitman, D., Northup, J., Hupp, S., y Murphy, M. (2003). Promoting social skills generalization with ADHD-diagnosed children in a sports setting. Behavior Therapy, 34, 313-330.

Pardos, A., Fernández, A., y Fernández, D.M. (2009). Habilidades sociales en el trastorno por déficit de atención/hiperactividad. Revista de Neurología, 48(2), 107-111.

Pelham, W.E., Massetti, G.M., Wilson, T., Kipp, H., Myers, D., Standley, B.B.N., ... y Waschbusch, D.A. (2005). Implementation of a comprehensive schoolwide behavioral intervention: The ABC Program. Journal of Attention Disorders, 9(1), 248-260.

Pelham, W.E., Wheeler, T., y Chronis, A. (1998). Empirically supported psychosocial treatments for attention deficit hyperactivity disorder. Journal of Clinical Child and Adolescent Psychology, 27(2), 190-205.

Pfiffner, L., y McBurnett, K. (1997). Social skills training with parent generalization: Treatment effects for children with attention deficit disorder. Journal of Consulting and Clinical Psychology, 65(5), 749-757.

Presentación, M., Siegenthaler, R., Jara, P., y Miranda, A. (2010). Seguimiento de los efectos de una intervención psicosocial sobre la adaptación académica, emocional y social de niños con TDAH. Psicothema, 22(4), 778-783.

Reitman, D., O'Callaghan, P., y Mitchell, P. (2005). Parent as Coach: Enhancing Sports Participation and Social Behavior for ADHD-Diagnosed Children. Child \& Family Behavior Therapy, 27(2), 57-68.

Sim, L., Whiteside, S. Dittner, C., y Mellon, M. (2006). Effectiveness of a Social Skills Training Program with School Age Children: Transition to the Clinical Setting. Journal of Child and Family Studies, 15, 409-418. 
Sonuga-Barke, E.J., Thompson, M., Abikoff, H., Klein, R., y Brotman, L.M. (2006). Nonpharmacological interventions for preschoolers with ADHD: the case for specialized parent training. Infants \& Young Children, 19(2), 142-153.

Storebo, O., Gluud, C., Winkel P., y Simonsen, E. (2012). Social-skills and parental training plus standard treatment versus standard treatment for childrenwith ADHD - The randomised SOSTRA trial. Plos One, 7, 1-8.

Stahl, L.C. (2007). The Social Goals of Boys with ADHD: Comparing Self-report and Peer Inferred Data (Doctoral dissertation, Pepperdine University).

Webster-Stratton, C. (2011). The incredible years: parent, teacher, and child training series (IYS). In Preventing violence and related health-risking social behaviors in adolescents: An NIH State-of-the-Science Conference (p. 73).

Webster-Stratton, C., Reid, J., y Hammond, M. (2001). Social skills and problem-solving training for children with earlyonset conduct problems: who benefits? Journal of Child Psychology and Psychiatry, 42, 943-952.

Wilkes, S., Cordier, R., Bundy, A., Docking, K., y Munro, N. (2011). A play-based intervention for children with ADHD: A pilot study. Australian Occupational Therapy Journal, 58, 231-240. 